\title{
Intertemporal Choice and Inequality
}

\section{Angus Deaton and Christina Paxson}

\author{
Princeton University
}

The permanent income hypothesis implies that, for any cohort of people born at the same time, inequality in both consumption and income should grow with age. We investigate this prediction using cohort data constructed from 11 years of household survey data from the United States, 22 years from Great Britain, and 14 years from Taiwan. The data show that within-cohort consumption and income inequality measures do indeed increase with age in the three economies and that the rate of increase is similar in all three. According to the permanent income hypothesis, the increase in inequality reflects cumulative differences in the effects of luck on consumption. Other models of intertemporal choice-such as those with strong precautionary motives or liquidity constraints-can limit or even prevent the spread of inequality, as can insurance arrangements that share risk across individuals. The evidence on the spread of inequality can therefore be used to help quantify the extent to which private and social arrangements moderate the impact of risk on the distribution of individual welfare.

\section{Introduction}

Suppose that the permanent income hypothesis $(\mathrm{PIH})$ is true, so that the consumption of each person in the economy follows a random

We are grateful to James Banks and Richard Blundell for the cohort data for Great Britain and to Orazio Attanasio for the U.S. data that were used in an earlier version of this paper. We also thank Tim Besley, Anne Case, Ben Eden, Steve Davis, Marjorie Flavin, Robert Hall, Alan Krueger, Robert Lucas, Thierry Magnac, Jean-Marc Robin, Joe Stiglitz, participants in the National Bureau of Economic Research's February 1993 Economic Fluctuations Research meeting, and an anonymous referee for helpful suggestions. We should like to thank the Lynde and Harry Bradley Foundation for financial support, and David Wise and the NBER Aging Program for starting us thinking about these issues. The first draft of this paper bore the title "Intertemporal Choice and Consumption Inequality." 
walk. It follows that the cross-sectional dispersion of consumption within a fixed-membership group of people will increase over time, provided that innovations to consumption are not perfectly correlated among people within the group. Increasing consumption inequality is financed by an increasing dispersion in total income, defined as the sum of earnings and asset income. It is not, however, necessary that there be increasing dispersion in both of these components of total income, and rising consumption inequality will be observed even if the cross-sectional distribution of earnings is constant. Since these results apply to any group whose membership is fixed over time, consumption and income inequality within a cohort of individuals born in the same year should increase as the cohort ages.

The result that inequality should increase over time within cohorts may seem at odds with the general-and broadly correct-perception that economywide measures of income and consumption inequality change slowly if at all. However, the PIH does not necessarily imply that aggregate inequality should increase over time, provided that people do not live forever. Inequality is greater among older cohorts and less among the young, and since young people are continually replacing the old, there is no automatic presumption that overall dispersion should increase. The secular behavior of aggregate inequality depends on how assets are passed from one generation to the next and on what the age structure of the population is.

That the PIH implies that the variance of consumption should increase over time within cohorts was originally proposed by Benjamin Eden in private correspondence, and some of the more general propositions are implicit in Eden (1980). This insight is the starting point for the investigations reported in this paper, although our perspective is more general than the PIH. The result on increasing inequality can also be derived from a range of other models, some of which are generalizations of the PIH and some of which contradict it. The theoretical behavior of inequality within cohorts depends on people's attitudes toward risk and on the mechanisms that are available for sharing risks between people and periods. The $\mathrm{PIH}$ assumes that people have certainty equivalent preferences, allows them to lend and borrow as much as they want, and permits no direct sharing of risk between people, for example through state-contingent asset or insurance markets. Changing any of these assumptions will generally affect the way in which risk is filtered into consumption inequality, and one of the main reasons for measuring consumption inequality and its evolution is to help understand and calibrate the way in which the economy handles risk (see Townsend [1994], Atkeson and Lucas [1992], and Lucas [1992] for closely related analyses).

Our procedure in this paper is the reverse of the usual one: we 
start with the evidence and follow with the theory. In Section II, we use data from the United States, Britain, and Taiwan to examine how much the within-cohort distributions of consumption, income, and earnings disperse as cohorts age. For each country, we contrast changes over time in within-cohort inequality with changes in aggregate inequality, although it should be kept in mind that explaining movements in aggregate inequality is not a purpose of this paper. The data we use are drawn from 47 annual household surveys from the three countries. Because the surveys are large and because we use so many of them, it is possible to document the facts using largely graphical analysis; we do not require a tight theoretical structure to identify parameters for estimation. In Section III, we consider various theoretical models: the permanent income hypothesis, intertemporal allocation with risk aversion, buffer stock models of saving and consumption under borrowing constraints, and models with risk sharing and insurance between individuals. For each we derive the implications for the evolution of within-cohort inequality and relate the theory back to our empirical findings.

\section{Evidence}

\section{Data and Construction of Cohorts}

Our general procedure for the three countries is to use successive years of cross-sectional household survey data to follow cohorts of individuals or individual households through time. While the survey data are not longitudinal, so that it is never possible for us to follow individuals or households over time, we can follow cohorts of people through their randomly selected representatives in each survey. Cohorts are identified by their year of birth or, equivalently, by their age in a base year. To take Taiwan as an example, we can take those born in 1945, who were 31 years old in the first survey year (1976), and we can examine the distribution of consumption, earnings, and incomes for all 31-year-olds in the 1976 survey. This distribution is then compared with the distributions of consumption, earnings, and incomes for all 32-year-olds in 1977, those for all 33-year-olds in 1978, and so on, ending with the distribution for 45-year-olds in 1990 , the last survey year. Such procedures are for many purposes superior to the use of panel data. Sample attrition is not an issue, and summary statistics from the cohort distributions are more likely to be accurate than the individual data from panels. The propositions of this paper concern the dynamics of the distributions of consumption in successive years and do not require knowledge of the joint 
distributions over several years, information that could come only from panel data.

A major difficulty is that while income and earnings data are available at the level of the individual, consumption data are available only for households. We must therefore examine the evolution in the distribution of consumption over households and convert income and earnings to the same basis. The difficulty lies in the fact that, unlike individuals, households form and dissolve over time, so that when we track households labeled by the age of the household head, we cannot always be sure that we are sampling from the same population in successive years. In practice, we believe that the difficulties are likely to be severe only among older households, where death, living with children, or institutionalization means that surviving households are increasingly selected and presumably increasingly unrepresentative. There are similar difficulties among very young household heads, who are also unlikely to be representative of their cohort, many of whom are in full-time education or (in Taiwan for males) in the military. Even among middle-aged heads, divorce and remarriage will imply that the population of households with heads aged 40 in one year is not the same as the population of households with heads aged 41 in the next. In spite of the problems, we believe that working with households is preferable to any attempt to convert to individuals by using assignment rules to allocate expenditures to household members. Such assignment rules are inevitably arbitrary, and different assignment rules may produce quite different results.

The Taiwanese data come from the Personal Income Distribution Surveys, which have been collected each year since 1976 . We use 14 surveys, covering 1976-90 but excluding 1978. In 1976 and 1977, the sample sizes are a little over 9,000 households; from 1978, there are over 14,000 households in each survey. We exclude data from 1978, which was the first year of the expanded survey, because the income data contain a number of outliers. These outliers do not appear to affect the means but inflate measures of dispersion to levels that are clearly incompatible with the adjacent years. The survey design is described in Republic of China (1989). For income and consumption there is a single interview, at which questions are asked about major items of income and expenditure in the past year. A separate control sample of a small number of households keeps diaries of all items of income and expenditures throughout the year. These households are regularly visited by field inspectors to control quality, and the results are used to monitor (but not to alter) the results from the main survey. Consumption is defined as total expenditure on all goods (both durable and nondurable). For Taiwan it is relatively difficult to do what we do for the other countries and subtract out durable expendi- 
tures, and this difference between the countries should be borne in mind when interpreting the results. Income is after-tax income from all sources, whereas earnings (measured before tax) are defined to include business and farm income. Note that although this is the best that can be done and is certainly preferable to assuming that all business income is the return to physical assets, the procedure inevitably introduces a component of capital income into our measure of earnings. The age profiles of household incomes, earnings, consumption, and saving are presented in Deaton and Paxson (1993b), who also describe the changing demographic structure of households in Taiwan.

The Taiwanese surveys are sufficiently large for us to define cohorts for each year of age. We restrict ourselves to households with heads between the ages of 20 and 75 inclusive, so that after the exclusion of 1978, we have in total 784 "observations" on cohort-year pairs, each observation consisting of a distribution over the households in that cohort-year. The number of households in each cohort-year varies depending on the year (with 1976 and 1977 smaller) and on the age of the household. Typical cells contain between 300 and 400 households, but because of selection out of the sample-for example, into the military, into an adult child's household, or because of death-cell sizes are smaller at young and old ages. For example, groups aged 20-25 have an average cell size of 119 households, and groups aged 70-75 have an average cell size of only 34 households. The cell sizes are large enough to compute fairly accurate measures of inequality. For example, the within-cell variances of the logarithm of consumption typically have standard errors equal to about 10 percent of their values for middle-aged households, and they increase to about 16 percent for those aged $20-25$ and to 26 percent for households aged 70-75.

The U.S. data come from the 1980-90 versions of the Consumer Expenditure Survey (CEX). The surveys sample between 5,700 and 8,300 households per year, with about a fourth of households surveyed in each quarter. We use urban households only; the CEX in 1982 and 1983 had no rural households, and for consistency we work with urban households in all periods. There are 14 cohorts defined by 5-year age bands, from those aged 11-15 in 1980 to those aged $76-80$ in 1980. The "age" of a cohort is defined as the midpoint of the 5-year band, and our sample includes households with heads aged 23 (e.g., 21-25) in 1980 to those aged 78 (e.g., 76-80) in 1990. So, for example, the youngest cohort of those aged 11-15 in 1980 is not included in the sample until 1990, when the household heads in this cohort are aged 21-25 and are assigned an age of 23. Likewise, the older cohorts drop out of the sample in later years. Households are 
allocated to the quarter in which they were interviewed so that, when we account for the fact that not all cohorts are observed in all years, there are 496 observations (an observation consists of a cohort-yearquarter cell). The numbers of households in each cell are in most cases between 200 and 400, with a minimum cell size of 103 for the cohort aged 78 in 1985. There is a good deal less attrition among older households in the United States than in Taiwan, because the elderly in the United States are more likely to continue as independent households than to live with their eldest son, as is typical (although decreasingly so) in Taiwan. The standard errors of within-cell inequality measures are similar to those for Taiwan, discussed above, but with smaller increases at old and young ages.

For the United States, household consumption refers to nondurable and nonmedical expenditures over the last quarter. Medical expenditure is excluded because many of the items in this category are subject to top-coding. Including medical expenditure has extremely little effect on the results that follow. Disposable income refers to the total after-tax income of the household in the last year, and earnings to before-tax labor income, again in the last year. Both income and earnings are subject to top-coding in the CEX. Top-coding affects only a small percentage of households (less than 1.3 percent in any year for income), but the prevalence of top-coding does increase slightly as households approach retirement age. We use the income and earnings figures provided by the survey and make no attempt to predict "true" income and earnings for top-coded households. Our treatment of top-coded values will tend to underestimate the increase in income and earnings inequality with age, something that one must keep in mind when interpreting the results.

The British data come from the Family Expenditure Survey, an annual survey that has been in continuous operation since 1954 collecting data on some 7,000 households each year. The sample is representative of Great Britain, which is the United Kingdom excluding Northern Ireland. We use 22 years of data, from 1969 to 1990, once again split into quarters by date of interview. We use information for 11 cohorts, in 5-year age bands, from those aged 5-9 in 1969 to those aged 55-59 in 1969. We restrict our analysis to those between the ages of 22 and 77 (age is defined as the midpoint of the 5-year group). If we allow for the fact that the youngest cohorts are not observed in the earliest years, nor the oldest in the latest years, there are 844 quarterly observations on the cohorts. The number of households in each of these cohorts varies from 56 (the 20-24 group in 1969) to 234 (the 33-37 group in 1982), with most cells containing 150-200 households. For the British data, consumption excludes durables and is reported on a weekly basis; earnings is the answer to a question 
about "normal weekly earnings"; and income is total disposable income, again with a weekly reference period. These data were kindly provided to us in cohort form by Richard Blundell in London. In consequence, there are a few cases in which we report calculations from the raw data for only Taiwan and the United States.

\section{Within-Cohort Age Profiles of Consumption Inequality}

We begin by examining the lifetime profiles of the variances of the logarithms of consumption. There are a number of reasons to work with the variances of logs. A model of autarkic intertemporal allocation with isoelastic preferences predicts that the variance of log consumption will be constant in the absence of idiosyncratic shocks, and this is a natural baseline from which to look for the dispersion that should occur under individual uncertainty. In the simple case of the $\mathrm{PIH}$ in which individual consumption paths are independent random walks, the consumption distribution at $t+1$ is the distribution at $t$ plus noise, so that each consumption distribution will be stochastically dominated by any earlier one. As a result, any summary measure of inequality that respects the principle of transfers will increase over time (Atkinson 1970). While it is possible to construct examples in which the variance of logs responds perversely to transfers at the very top of the distribution, this is more a theoretical curiosity than a good reason to abandon such an otherwise convenient measure.

Figure 1 for Taiwan, figure 2 for the United States, and figure 3
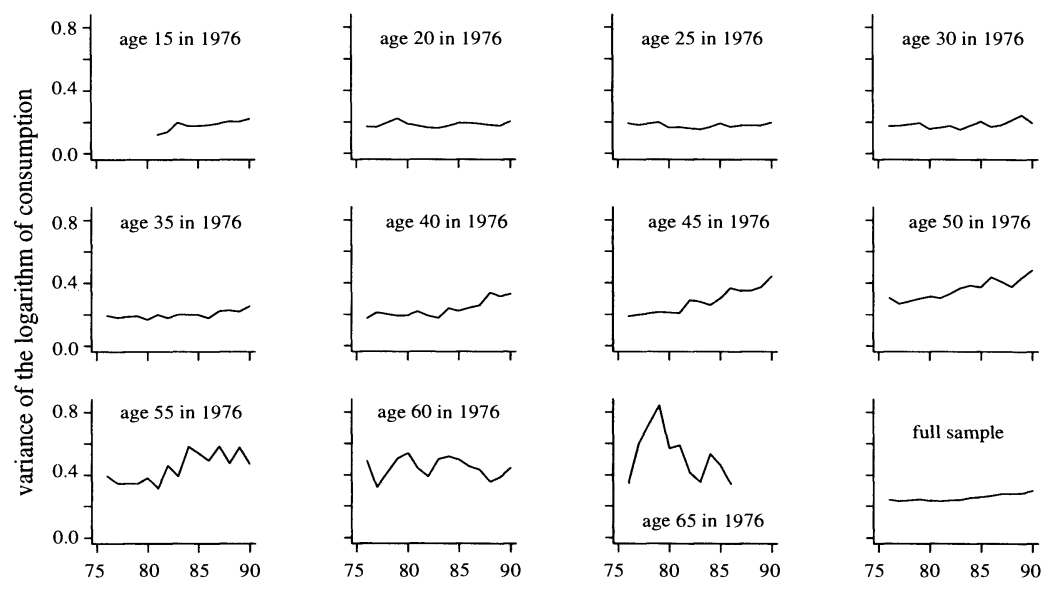

year

FIG. 1.-Taiwan: variances of log consumption and age, selected cohorts 

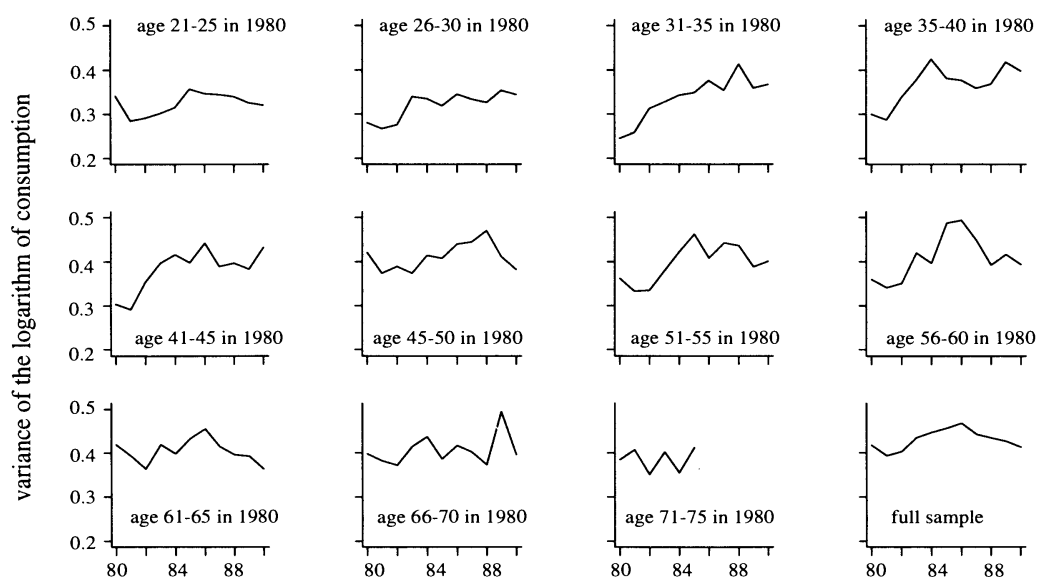

year

FIG. 2.-United States: variances of log consumption and age, all cohorts
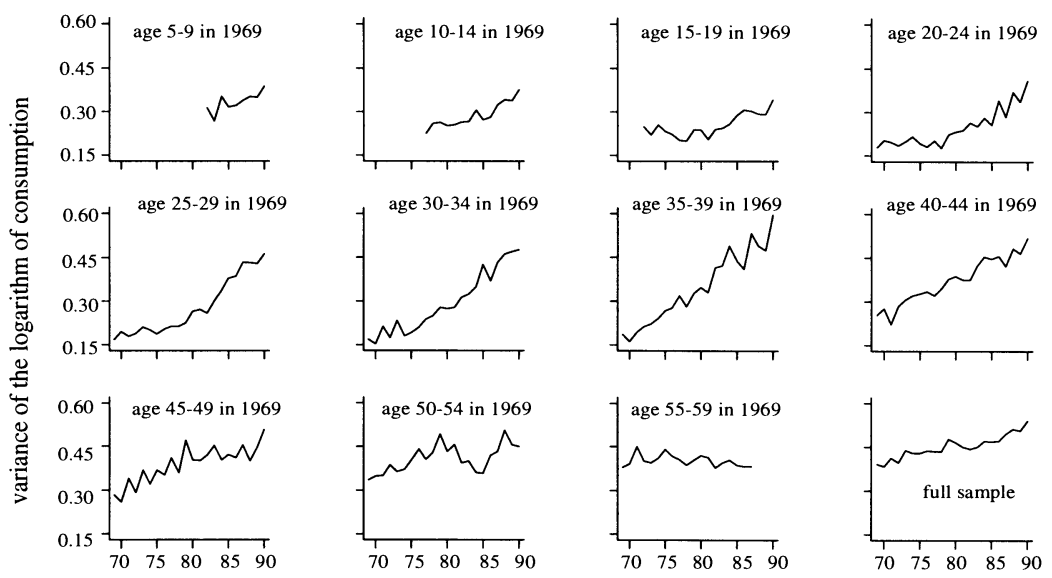

year

Frg. 3.-Great Britain: variances of log consumption and age, all cohorts

for Britain show profiles of within-cohort consumption inequality. Each panel shows the experience of a single cohort. The horizontal axis denotes the year of observation (as well as age of household head, given that each graph follows a single cohort), and the vertical axis is the variance of log consumption. For Taiwan, where we have too many cohorts to illustrate in full, we show the graphs for every fifth cohort. For the United States, we omit graphs for the oldest and the two youngest cohorts, which are not in the sample in all years. 
The figures shown in the graphs are the raw variances for Taiwan, but for the United States and Britain, where the surveys have a quarterly basis, seasonal variation in inequality is removed by averaging over the four quarters of each survey year. The final panel in each graph shows aggregate inequality and plots the variance of log consumption for all households in each survey year. Because overall inequality is a weighted average of within-cohort inequality plus inequality across cohorts, aggregate inequality is typically higher than inequality for individual cohorts. In Taiwan, however, the crosssectional age profile of consumption is very flat (see Deaton and Paxson 1993a), producing little across-cohort inequality, and overall inequality is approximately the weighted average of the within-cohort inequalities.

In all the countries, the inequality of consumption increases with age, although not linearly. In Taiwan, there is little spread of consumption inequality before age 40 , and the maximal dispersion appears to have been reached by age 60 . In both the United States and Britain, dispersion starts earlier, although once again there is a slowing down after age 60 . The age patterns are clearer in figure 4 , which uses data for all cohorts within the age cutoffs and filters out cohort effects and noise. These graphs are obtained by regressing the raw variances on a set of age, cohort, and (for the United States and Britain) quarter dummies. The graphs display the estimated age effects. The vertical position of each is chosen so that at a specific age-38 in Taiwan, 36-40 in the United States, and 35-39 in Brit-
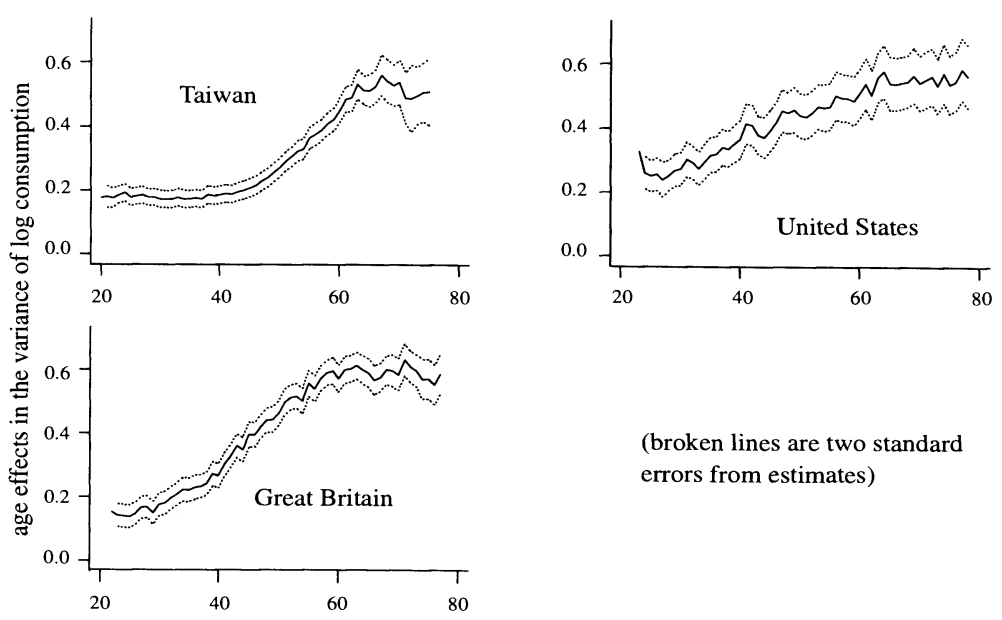

(broken lines are two standard errors from estimates)

Fig. 4.-Age effects (and confidence bands) for the variance of log consumption 
ain-the graphs go through the actual inequality for that age group averaged over all the survey years. (It is possible to add year dummies to these regressions, provided that a suitable normalization is adopted to deal with the linear dependency between year, cohort, and age effects first discussed in Hall [1971]; Deaton and Paxson [1993b] propose a suitable normalization, and the application of that method leads to results almost identical to those displayed in the figure.)

Figure 4 also shows as dashed lines confidence intervals for the estimated age effects. For the United States and Taiwan, the standard errors on which they are based have been adjusted to account for the fact that the inequality measures are based on samples drawn from each cohort. Since these regressions have dummies on the right-hand side, the effect of the sampling is to add what is effectively measurement error to the dependent variables. Because the cohort sizes differ by age and year, these measurement errors are heteroskedastic, but their variances can be estimated from the surveys and used to correct the ordinary least squares standard errors. For Taiwan, the adjustment increases the standard errors for the oldest age groups, where the cohort sizes are small, relative to the younger age groups, where they are larger. The adjustment makes little difference for the United States, where there is much less variation in cell sizes. For Britain, we cannot adjust the standard errors since we do not have the raw data necessary to do so, but the results for the United States suggest that adjustment would make little difference.

The age profile of inequality for the United States is close to linear, although the rate of increase of dispersion slows down slightly after age 60. In Taiwan, the profile is convex, and in Britain, slightly so; in both these countries, there is no further widening of consumption inequality after age 60 . The scale of the three panels in figure 4 is the same, and they show that the total increase in the variance of log consumption over the life cycle is very similar in all three countries, from about .2 to about .6, a little more in Britain and a little less in the United States. These numbers are confirmed in table 1, where the estimated age effects are regressed on age. The variance of logs within each cohort increases by .07 every decade in the United States, by .08 in Taiwan, and by .10 in Britain.

These age-related changes in consumption inequality are very large according to the standards whereby changes in inequality are usually assessed, as well as in comparison to any recent changes in inequality for the population as a whole. The last panels of figures 1, 2, and 3 show the variance of log consumption over all households for each of the survey years. For Taiwan, the last panel of figure 1 shows that the variance of log consumption over all households has increased from .24 in 1976 to .30 in 1990. Although this increase has been 


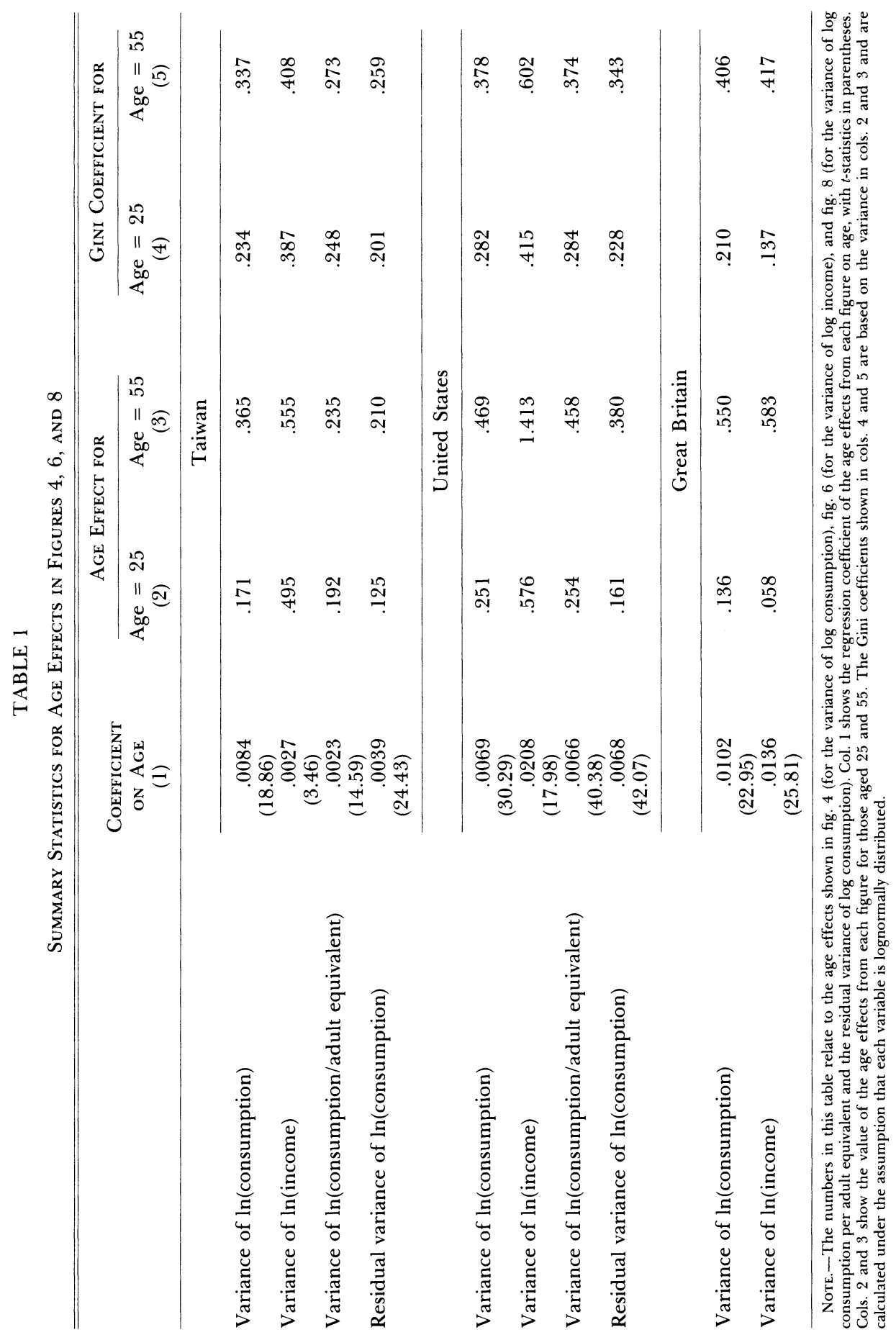


large enough to cause concern in a country that is perhaps the world's leading case of "growth with equity," it is small compared to the increase in within-cohort inequality with age. In fact, the increase in aggregate inequality in Taiwan may be linked to the increase in within-cohort inequality with age (see Deaton and Paxson 1993a). Demographic change has "aged" the Taiwanese population, increasing the share of older (and more unequal) households in the population relative to the share of younger (and more equal) households and producing higher aggregate within-cohort inequality. In the United States, the variance of log consumption was .42 in 1980 , rose to a maximum of .47 in 1986, and had fallen back to .41 by 1990 . The increase in the early 1980s was again large enough to generate concern and a good deal of literature (see Cutler and Katz [1991] and Levy and Murnane [1992], who also review other studies). Although the increase in U.S. inequality was clearly not due to population aging, as in Taiwan, it is very small compared with the size of the effect of aging on the within-cohort variance. The last panel of figure 3 indicates that aggregate inequality increased in Britain over the sample period. The aggregate variance of log consumption rose from .39 in 1969 to .54 in 1990. Although large, this increase is still small relative to the increase in within-cohort inequality with age. Table 1 converts changes in within-cohort inequality with age to Gini coefficients under the illustrative assumption that the distributions of consumption are lognormal. Once again, the changes are large. In the United States, where they are the smallest, the Gini increases from .282 at age 25 to .378 at age 55 , a change that should be compared with the increase in the Gini of consumption over all households, which rose from .355 in the first quarter of 1980 to .399 at its maximum in the third quarter of 1986.

The results discussed above are not merely due to our choice of the variance of logs, and any measure of consumption inequality that preserves the principle of transfers will generally increase over time within cohorts. This is demonstrated for Taiwan in figure 5, which shows what are essentially Lorenz curves for nine of the cohorts in the years 1976, 1983, and 1990. Because standard Lorenz curves are typically too close together to permit a useful visual comparison of the distributions, we plot their complements. The graphs show the vertical difference between the 45-degree line and the Lorenz curve, so that the cumulative fraction of the (ordered) population on the horizontal axis is plotted against, on the vertical axis, the same cumulative fraction of population less their cumulative fraction of total consumption. Like the Lorenz curves themselves, these curves indicate stochastic dominance, so that if the two lines do not cross, the 

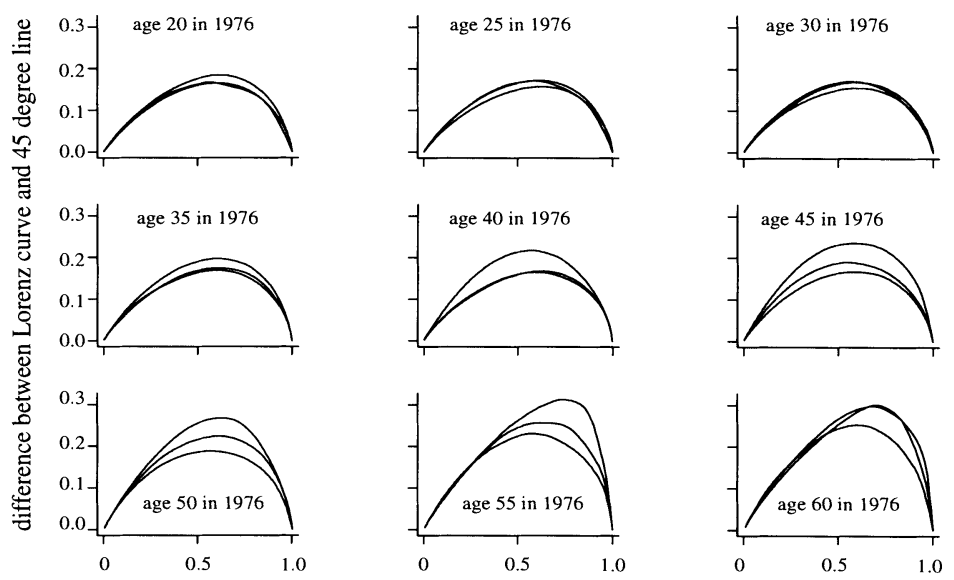

cumulative fraction of population

Fig. 5.-Taiwan: dispersion in consumption in 1976, 1983, and 1990

higher curve has more inequality according to any transfer-respecting inequality measure.

Figure 5 shows clearly not only the higher inequality among the older cohorts but also that (with a few doubtful calls) the lines for 1990 are above the lines for 1983 , which are in turn above the lines for 1976. Of course, graphs of Lorenz curves for all the years would show more crossing and declines in inequality between some pairs of successive years, just as the cohort plots of the variance of the log of consumption in figure 1 sometimes decline from one year to the next. Lorenz curves for the United States (not shown) are similar, although the picture is complicated by the inequality "boom" that affected all cohorts in the mid-1980s. Because the boom affected intracohort inequality as well as intercohort inequality, there are several cohorts whose "complementary" Lorenz curves are sometimes higher in the mid-1980s than in either 1980 or 1990 . However, if we take the years 1980 and 1990, where aggregate inequality is the same, we once again see that the later curves are higher, so that on average we obtain the stochastic dominance result.

\section{Within-Cohort Age Profiles of Inequality in Earnings and Income}

Whatever the explanation for the dispersion of consumption, a link with income or earnings or both is an obvious possibility, although as we shall see in the theoretical section, the same evidence can be interpreted in several different ways. In this subsection we present 
evidence on changes in income and earnings inequality with age. As was done for consumption, we regress the variances of the logarithms of income and earnings on sets of age, cohort, and (for the United States and Britain) quarter dummies. Figure 6 graphs the age effects from these regressions, superimposed on the age effects in the variances of log consumption from figure 4 . Age effects for the variance of log earnings are graphed only up to age 65 , after which the results are dominated by retirement and selection effects. Since the dispersions of income and earnings are typically much larger than that of consumption-except for Britain, perhaps because of the weekly reporting of consumption contrasting with "normal" earnings and income-it is no longer possible to maintain the same scale for the three countries. In particular, the rise of the consumption variance with age in the United States is now masked by the very much larger levels and rates of increase in income and earnings inequality.

The figure shows that, like consumption, earnings and income disperse with age. These results are not the product of using the variance of logarithms, nor are they determined by the regression procedures used to construct the figure. The fuller analysis applied to consumption in figures $1-5$ gives similar results for earnings and for income. Although all three countries show increasing dispersion in income with age, the relationship between the age effects in consumption and income differs across the three countries. For Taiwan, the predicted increase in the variance of log income (from age 25 to age

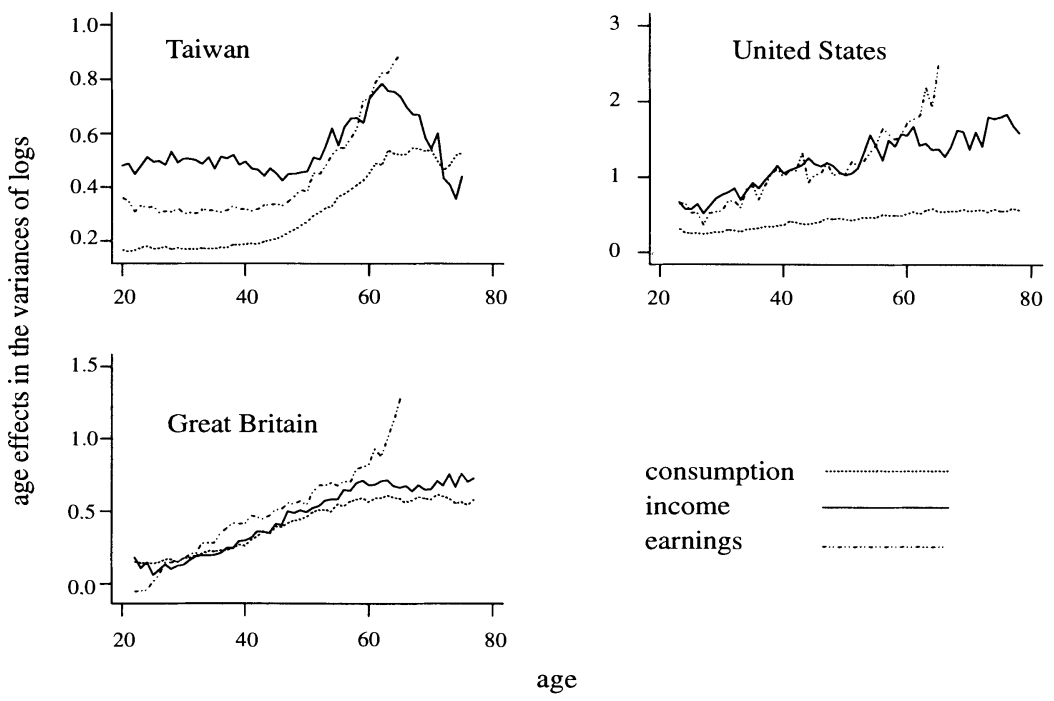

FIG. 6.-Age effects in the variances of logarithms of income, earnings, and consumption. 
55 ) is about one-third the size of the increase in the variance of log consumption (see table 1). In the United States, the variance of income increases much more rapidly with age than the variance of consumption, and in Britain the two variances show similar increases. The patterns of dispersion in income and earnings also differ somewhat across countries. In the United States and Britain, the variance of earnings is close to the variance of income, as it must be given that most households in both countries have little or no asset income. The result for earnings in the United States is consistent with previous examinations of individual earnings variances, such as Dooley and Gottschalk (1984), who use the Current Population Survey for 196879 to examine the variances of earnings within experience cohorts of men. The results for Britain are particularly suggestive of the simplest model, where consumption, earnings, and income are approximately equal. When the graphs for earnings and income diverge, as they do among households headed by older people, the results should not be treated too seriously because of the selection out of the labor force among the elderly. In Taiwan, where households saved more than 20 percent of their incomes during the 1980s, capital income is an important component of household disposable income, and increasingly so with age, so that earnings and incomes are a good deal further apart than in the United States and Britain. Even so, their patterns of dispersion are similar, and both mimic the convex pattern of consumption dispersion. However, we must again emphasize the fact that we have designated income from small business as earnings in Taiwan, so that the division between earnings and income is far from firmly based. The rapid fall in income variance among the elderly in Taiwan should also be seen in the light of the much more severe selection against households with elderly heads in that country.

\section{Household Size and Composition}

One essentially mechanical explanation for an increasing dispersion of household consumption is that there is an increasing dispersion of household size and that consumption-and presumably also income and earnings - is tied to household size and composition, and so will disperse in parallel with it. While we would expect some such effect, we are concerned to establish that there is within-cohort dispersion with age beyond what can be explained in this obvious way. We investigate this issue only for the United States and Taiwan, for which we currently have the raw data in hand.

The left-hand panels of figure 7 show the age profile of the number of adult equivalents in Taiwan and the United States, defined as the number of household members over 17 years of age plus half of 

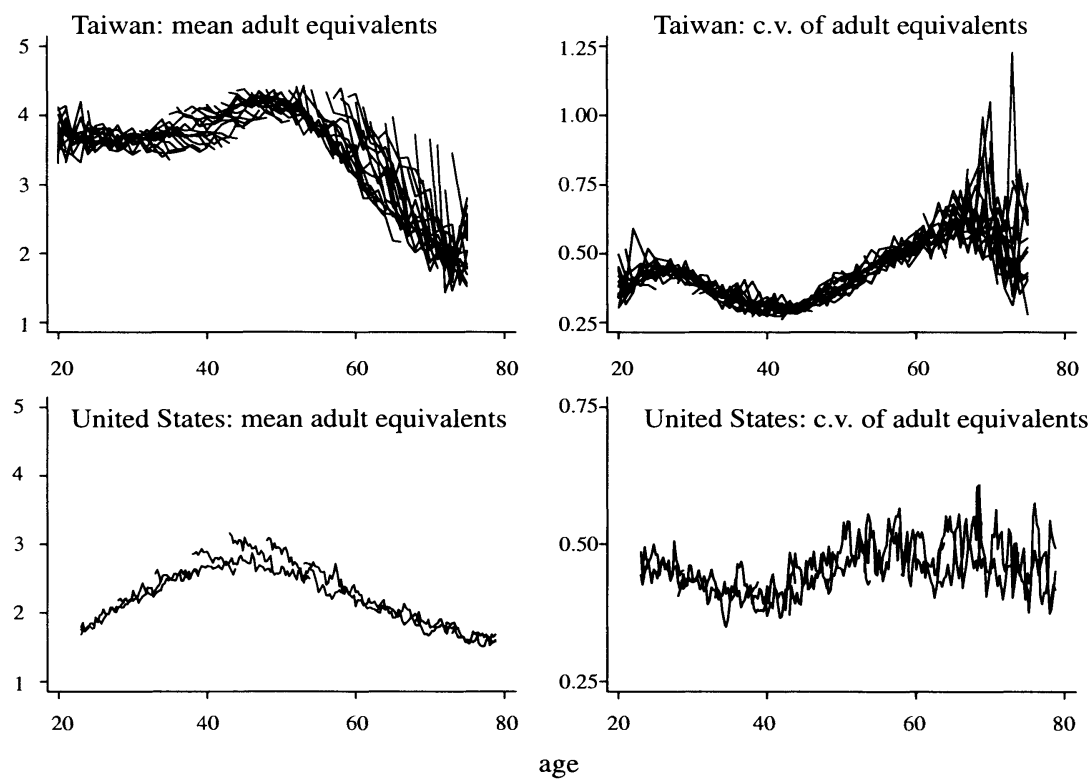

Fig. 7.- Mean and coefficient of variation of adult equivalents for each cohort by age, Taiwan and the United States.

the number aged 17 or less. Each continuous line shows the average numbers for a given cohort over time, and the figure shows the characteristic life cycle pattern of household size together with (in Taiwan) the substantial cohort effects associated with the decline in fertility over time. The apparent variability among the older cohorts is a sampling effect, since there are few households in these cohorts over which to calculate the means. The right-hand panels show the coefficient of variation of adult equivalents for the same cohorts. The point to note here is that for both countries from ages 40 to 60 , although average household size is falling for most of the period, the dispersion of household sizes is rising. This increase is much larger for Taiwan than for the United States (note that the scales for the vertical axis differ for the two countries). It is likely that some of the rising variability of total household consumption that we have already documented may be attributable, not to the accumulated effects of idiosyncratic uncertainty, but to the fact that dispersion of household sizes increases in the critical age range.

Figure 8 illustrates the results of two simple experiments designed to correct consumption inequality for household size effects. In the first, shown by the upper (broken) line in each of the panels, we show the age effects corresponding to those in figure 4, but for the variance of logarithms of consumption per adult equivalent as opposed to total 

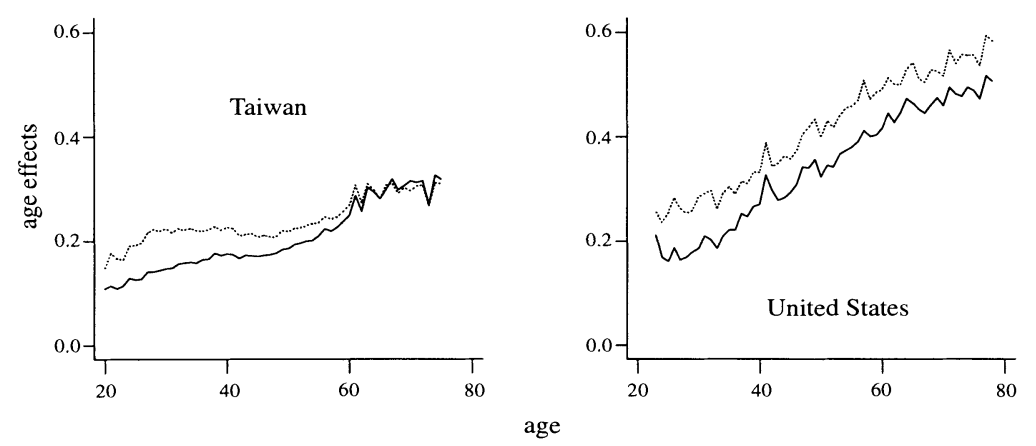

variance of log consumption per adult equivalent residual variance of $\log$ consumption

Fig. 8.-Age effects in the variance of log consumption adjusted for household size and composition.

household consumption. The lower (solid) lines show age effects in the variance of the residuals of regressions (estimated separately for each cohort-year or cohort-year-quarter cell) of the logarithm of household consumption on a range of demographic variables. Both methods give similar results. In Taiwan, the dispersion of household size can account for a good deal of the dispersion in household consumption. For example, table 1 shows that when consumption is put into adult equivalent form, the predicted variance of log consumption rises by .043 between the ages of 25 and 55 , in contrast to an increase of .194 when consumption is not adjusted for household size. In the United States, where the dispersion of sizes is much smaller, the dispersion of consumption with age is hardly affected by adjustment for household size. In both cases, there is dispersion of consumption with age that is not accounted for by any simple link to household size.

\section{Theoretical Interpretations}

As we outlined in the Introduction, the permanent income hypothesis implies that within-cohort consumption and income streams will fan out as the cohort ages, and the testing of this prediction motivated our research in the first place. In this section, we justify and fill out our theoretical claims and show how the PIH can be used to make a number of other predictions about the evolution of inequality. The PIH turns out to be remarkably consistent with the empirical evidence on dispersion, although as we shall see as we discuss the other theories, it is by no means the only model capable of explaining the facts. 
Indeed, there is a great deal of other evidence that casts doubt on the PIH-reviewed, for example, in Deaton (1992)-so that our aim here is to add to the theoretical and empirical understanding of the PIH rather than to seek to defend it as an adequate explanation of consumption behavior in general. There is currently no one model of consumption that is both theoretically consistent and capable of explaining all the evidence, so we offer our results as another piece in the puzzle, a piece that will have to be fitted into whatever final synthesis is eventually reached.

\section{Inequality and the Permanent Income Hypothesis}

We begin by showing that the PIH implies that consumption inequality within cohorts will increase over time. Suppose that preferences are intertemporally additive, that each subperiod's quadratic utility function is identical up to discounting by the rate of time preference, and that the real interest rate is constant and equal to the rate of time preference. Then optimal intertemporal choice implies the PIH, and consumption follows a martingale (Hall 1978),

$$
c_{i t}=c_{i t-1}+u_{i t},
$$

where $i$ indexes the individual (or household) and $t$ the time period. Assume also that, at any given time $t$, the cross-sectional covariance is zero:

$$
\operatorname{cov}\left(c_{i t-1} u_{i t}\right)=0
$$

the validity of (2) will be discussed below. Using (1) and (2), we can take variances over any set of individuals in existence at both $\mathrm{t}-1$ and $\mathrm{t}$, to give

$$
\operatorname{var}_{t}(c)=\operatorname{var}_{t-1}(c)+\sigma_{t}^{2},
$$

where $\sigma_{t}^{2}$ is the period $t$ variance of $u_{i t}$. Equation (3) indicates that the variance of consumption increases over time. In addition, if (2) is strengthened to the assumption that the shocks $u_{i t}$ in (1) are independent of the consumption levels $c_{i t-1}$, equation (1) implies the stronger result that the cross-sectional distribution of consumption at $t$ is (second-order) stochastically dominated by the cross-sectional distribution of consumption in any earlier period. The Lorenz curve evidence for Taiwan in figure 5 is consistent with this prediction.

Note the requirement that the group of people whose variance is being computed is constant in both $t$ and $t-1$. The result on consumption inequality is true only for a group with fixed membership, not for the population as a whole, where old people are constantly being replaced by newborns. If people live forever or if families live 
forever through eternal dynasties, then (3) will imply that consumption inequality should be increasing over time for society as a whole. The relative constancy of aggregate inequality in the final panels of figures 1,2 , and 3 would therefore seem to be evidence against the dynastic version of the PIH.

The result that within-cohort consumption inequality increases through time requires several caveats. First, there are limits on this process set by the limitations of the PIH and the random walk model itself. Presumably consumption will not become negative or exceed the bliss point in quadratic utility. Second, the result that inequality increases could be either strengthened or weakened if we were to allow either for subperiod utility functions that differ over time (socalled taste shifters) or for heterogeneity in preferences across individuals. Homogeneous tastes within the PIH imply that, with no uncertainty, each individual's consumption level will be constant so that there is no increase in inequality with age. In a stochastic environment, increases in inequality are due solely to the accumulated effects of idiosyncratic uncertainty. Heterogeneity in preferences across individuals or taste shifters for individuals over time may be an additional source of changes in inequality, but without restriction, heterogeneity can be made to generate any pattern of consumption dispersion over time, including decreases as well as increases. The evidence in figure 8 indicates that one of the most important sources of heterogeneityvariations in household size-can account for a large part (but not all) of the observed increase in consumption dispersion for Taiwan, although not for the United States.

A final caveat concerns the assumption in (2), that the crosssectional covariance between $c_{i t-1}$ and $u_{i t}$ is zero. It is important to note that this assumption is not an implication of the PIH. Although the PIH implies that, for each individual $i, u_{i t}$ is orthogonal to previously known information, including $c_{i t-1}$, the PIH does not imply that (2) has to be true in the cross section (see Chamberlain 1984). However, if we make the additional assumption that lagged aggregate consumption is in each person's information set, then the expectation over time of the cross-sectional covariance between $c_{i t-1}$ and $u_{i t}$ will be zero. To see this, write $\theta_{t}$ for the covariance in (2), so that

$$
\theta_{t}=E\left[\left(c_{i t-1}-c_{t-1}\right)\left(u_{i t}-u_{t}\right) \mid t\right]=E\left(c_{i t-1} u_{i t} \mid t\right)-c_{t-1} E\left(u_{i t} \mid t\right),
$$

where variables with only $t$ subscripts are averages over households, and the explicit conditioning on $t$ is to emphasize the fact that these are cross-sectional expectations. Take expectations of (4) over time and use the law of iterated expectations to give

$$
E\left(\theta_{t}\right)=E\left[E\left(c_{i t-1} u_{i t} \mid i\right)\right]-E\left[E\left(c_{t-1} u_{i t} \mid i\right)\right] .
$$


The terms inside the brackets relate to individual behavior over time, about which the theory is informative. The first term is zero, because individual innovations are orthogonal to individual lagged consumption levels. The second term will be zero if we assume that lagged macro consumption is known to each individual. This is a standard assumption in aggregating Euler equations-see particularly Grossman and Shiller (1982) - but it is by no means obviously correct, and its failure can perhaps account for some of the rejections of the PIH in macro data (see Pischke [1991], Goodfriend [1992], and Deaton [1992, chap. 5] for a review). However, if we accept the assumption that people are informed about aggregate consumption, we have the result that although consumption dispersion need not increase in every period, it can be expected to do so on average. This prediction is consistent with the experience in the United States through the 1980 s. Although within-cohort consumption inequality generally increases with age, the inequality boom in the mid-1980s temporarily distorts the pattern.

The PIH implies that consumption inequality increases with age, no matter what stochastic process governs the evolution of earnings. However, the shape of the age-inequality profile depends on the earnings process. To examine this relationship, it is useful to first derive an explicit formula linking the dispersion of consumption to innovations in earnings. As usual with the PIH, suppose that there is a single real asset $A_{t}$ that evolves according to

$$
A_{t}=(1+r)\left(A_{t-1}+y_{t-1}-c_{t-1}\right),
$$

where $y_{t}$ is earnings in period $t$ and $r$ is the constant real interest rate; we have temporarily suppressed the individual subscripts $i$. This equation, together with the random walk (1) and a condition that assets be zero in a terminal period $T$, yields the finite-life version of the PIH:

$$
\beta_{t} c_{t}=\frac{r}{1+r} A_{t}+\frac{r}{1+r} \sum_{k=0}^{R-t}(1+r)^{-k} E_{t} y_{t+k},
$$

where $\beta_{t}$ is an annuitization factor $1-\left[1 /(1+r)^{T-t+1}\right]$. When $T$ is infinite, $\beta_{t}$ is unity, and ( 7$)$ is the standard infinite horizon PIH as in Flavin (1981); otherwise it is a concave and monotone declining function of $t$, with values close to unity at the beginning of life and declining to $r /(1+r)$ at death. The variable $R$ is the retirement date, after which earnings equal zero.

If (6) is used to substitute for assets in (7) and the first lag of (7) is subtracted from the result, we obtain the martingale property of consumption in the explicit form

$$
\beta_{t} \Delta c_{t}=\eta_{t},
$$


where $\eta_{t}$ is a consumption innovation, related to the innovations in earnings by

$$
\eta_{t}=\frac{r}{1+r} \sum_{k=0}^{R-t}(1+r)^{-k}\left(E_{t}-E_{t-1}\right) y_{t+k} .
$$

We can rewrite (9) in terms of the history of the innovations, so that

$$
c_{t}=c_{0}+\sum_{\tau=0}^{t} \beta_{\tau}^{-1} \eta_{\tau},
$$

so that, since the innovations are serially uncorrelated,

$$
\operatorname{var}\left(c_{t}\right)=\operatorname{var}\left(c_{0}\right)+\sum_{\tau=0}^{t} \beta_{\tau}^{-2} \sigma_{\eta \tau}^{2},
$$

where $\sigma_{\eta t}^{2}$ is the variance of age $t$ 's consumption innovation.

Equations (8)-(11) have several implications for the shape of the age-inequality profile. First, if there are no earnings after retirement, there can be no further innovations in consumption, and there will be no further dispersion in the cross-section distribution of consumption. This is exactly the result in figure 4 for Taiwan and Britain, but not for the United States. Of course, in any given cohort, not everyone will retire on the same date, and there are also likely to be idiosyncratic surprises in rates of return that will result in idiosyncratic innovations to consumption even after retirement. Even so, the PIH certainly predicts that the rate of growth of consumption inequality will at least slow down among old cohorts. One possible explanation for the apparently anomalous behavior of the United States is uncertainty regarding health care costs, which are covered by the state in Britain and are very small in Taiwan. However, the U.S. consumption measures used above exclude health care costs, and including them makes no appreciable difference to figure 2 or 4 .

Second, whether the age-inequality profile is concave or convex (up to the age of retirement) depends on the degree of persistence in shocks to earnings. Specifically, the age-inequality profile will be concave unless individual earnings contain a large stationary component. To see this, first consider the case of white-noise earnings with $\eta_{t}=$ $r \epsilon_{t} /(1+r)$, where $\epsilon_{t}$ is the shock to earnings. In this case, the terms $\sigma_{\eta t}^{2}$ in equation (11) do not vary with age. Since $\beta_{t}^{-2}$ is increasing and convex in $t$, the variance of consumption must also increase at an increasing rate. With white-noise earnings, earnings shocks have no 
implication for the future, and their effects on consumption are spread over a larger number of years for younger consumers. However, if earnings are persistent, $\sigma_{\eta t}^{2}$ will decline with age, possibly enough to yield a concave age-inequality profile. For example, consider the case in which the first difference of earnings is a first-order moving average $\epsilon_{t}-\theta \epsilon_{t-1}$, with $1 \geq \theta \geq 0$. This occurs if earnings are the sum of a random walk and white noise, and it collapses to the white-noise case if $\theta=1$. In this case (8) and (9) imply that

$$
\Delta c_{t}=\beta_{t}^{-1}\left[\beta_{t}^{R}(1-\theta)+\theta r(1+r)^{-1}\right] \epsilon_{t},
$$

where $\beta_{t}^{R}$ is the annuitization factor computed to $R$ instead of to $T$. The term inside the brackets decreases in age unless $\theta=1$, implying that the variances $\sigma_{\eta t}^{2}$ also decline with age. The age-inequality profile will be concave if the full term multiplying $\epsilon_{t}$ in (12) decreases in age. This will be true if

$$
\frac{1}{\theta}>1+\frac{r}{(1+r)\left[(1+r)^{T-R}-1\right]},
$$

which holds for reasonable values of $r, T$, and $R$ unless $\theta$ is very close to one. Hence, unless the unit root has a very low share of the innovation variance in earnings, we can expect the age-inequality profile to be concave. Older households are closer to retirement and so get the benefits of an earnings innovation for fewer periods; they therefore spend less of it and save more.

The prediction of concave age-inequality profiles is not borne out for any of the countries, where consumption inequality is either linear (United States) or convex in age (Taiwan and Britain). These results in figure 4 imply that if the PIH is valid, earnings contain a large stationary component. On the other hand, the lack of concavity in the age-inequality profiles might cast doubt on the PIH interpretation of the data.

Our final result from the PIH concerns the dispersion of income. We show that an implication of the PIH is that the dispersion of income increases with age (up to retirement) and that the rate of dispersion depends on the stochastic process for earnings. Consider the identities

$$
y_{t}^{d}=\frac{r}{1+r} A_{t}+y_{t}=\beta_{t} c_{t}+s_{t},
$$

which define disposable income $y_{t}^{d}$ as the sum of asset income and earnings and saving $s_{t}$ as the difference between disposable income and $\beta_{t} c_{t}$. Saving is defined in this nonstandard way so that it satisfies 
Campbell's (1987) "rainy-day” equation

$$
s_{t}=\sum_{k=1}^{R+1-t}(1+r)^{-k} E_{t}\left(-\Delta y_{t+k}\right),
$$

whereby saving is the discounted present value of expected future falls in earnings. (This can be proved directly by substituting [7] into the last equality in [14] and rearranging.) Note that the expected falls in earnings in (15) include the fall in earnings that will take place immediately after retirement; indeed even if earnings are constant or are expected to remain so (a random walk), there will still be a role for retirement saving. From (14), the PIH (7), and the rainy-day equation (15), disposable income can be written in terms of earnings as

$$
y_{t}^{d}=\beta_{t}\left(c_{0}+\sum_{\tau=1}^{t} \beta_{\tau}^{-1} \eta_{\tau}\right)-\sum_{k=1}^{R+1-t}(1+r)^{-k} E_{t} \Delta y_{t+k} .
$$

For any given stochastic process for earnings, (16) yields the corresponding process for disposable income; for a group of people with identical earnings processes, it thus controls the dispersion of disposable income over time.

To analyze the rate at which disposable income disperses, we separate the cases of stationary and nonstationary earnings. In the former, the cross-section distribution of earnings will be constant, and there will be no dispersion with age in the within-cohort earnings distribution. From (15), saving, which is the sum of saving for retirement and short-run "smoothing" saving, is also stationary, and its cross-section distribution will be constant. Disposable income is the sum of consumption (which is an integrated process) and saving (which is stationary) and so is itself integrated, and its distribution will disperse at the same rate as that of consumption.

This is apparently not the case for any of the data in Section II, since for all three countries, the within-cohort earnings distributions are dispersing with age. Suppose then that earnings processes are nonstationary, so that earnings are dispersing as we see in the data. Even if the first difference of earnings is stationary, (15) implies that savings are nonstationary, because saving for retirement is (partly) determined by the expected value of earnings at retirement, which is itself nonstationary. Disposable income is thus the sum of two integrated processes, each with positive weights on the earnings innovations, so that once again the variance of disposable income will grow until retirement, but now more rapidly than the rate at which the 
variance of consumption expands. Assets are the sum of previous saving and so will be an $I(2)$ process, whose cross-sectional variance will therefore expand more rapidly than that of either consumption or disposable income. The evidence in figure 6 , whose interpretation is clouded by selection effects at higher ages, suggests that earnings and incomes disperse at about the same rate, which is indicative of nonstationary earnings but with little or no saving for retirement. This is surprising only for Taiwan, which is the only country of the three in which households save a substantial fraction of their incomes; but as we have demonstrated earlier (Deaton and Paxson 1993b), there are no strong age effects in Taiwanese household saving rates.

\section{Relaxing Certainty Equivalence}

We have dealt with the PIH first because it was our own starting point and because the algebra is simple enough to allow derivation of clear results. However, the assumptions underlying the PIH are very strong, and in this subsection we consider the implications of more general models of intertemporal choice for the dispersion of consumption.

With intertemporally additive preferences and a constant rate of time preference, the evolution of consumption is controlled by the Euler equation

$$
\left(1+r_{t+1}\right) \lambda\left(c_{i t+1}\right)=(1+\delta) \lambda\left(c_{i t}\right)+u_{i t},
$$

where $\lambda(c)$ is the instantaneous marginal utility (felicity) of consumption function and $r_{t+1}$ is the real rate of interest from $t$ to $t+1$. If impatience is large enough so that $\delta \geq r_{t+1}$, then the marginal utilities of consumption become more dispersed over time, in every period if the innovations are independent of lagged values in the cross section, otherwise on average. When the rate of interest is greater than the rate of time preference, the distribution of marginal utilities in the cross section can either concentrate or disperse, depending on the cross-sectional dispersion of the innovations.

The relationship between the dispersion of consumption and the dispersion of marginal utilities depends on the function $\lambda(\cdot)$. If the distribution of marginal utilities in $t$ is stochastically dominated by the distribution of marginal utilities in $t-1$, the same will be true for the distribution of consumption if the inverse function $\lambda^{-1}(\cdot)$ is concave. Since $\lambda(\cdot)$ is monotone declining, $\lambda^{-1}(\cdot)$ will be concave if and only if $\lambda(\cdot)$ is concave. We therefore have the result that the distribution of consumption in $t$ stochastically dominates the distribution of consumption in $t+1$ if $\delta \geq r_{t+1}$ and if the marginal utility of consumption function is concave. The condition that $\lambda(\cdot)$ be convex is 
what generates a precautionary motive for saving and has received a good deal of recent attention as a potential explanation for a number of empirical phenomena. The concavity of marginal utility generates the opposite of precautionary saving, an "antiprecautionary" motive, and is perhaps implausible. That a strong enough precautionary motive should inhibit the spread of inequality should not in itself be surprising; precautionary saving is motivated by the desire to minimize future consumption variability. As a result, "prudent" consumers will avoid the risks that are the fundamental cause of increases in inequality.

Impatience and concavity of marginal utility, although sufficient to guarantee increasing dispersion, are not necessary. Consider, for example, the case of isoelastic utility in which, with no uncertainty and identical preferences, each household's consumption will be growing at the same (positive or negative) rate. The variance of log consumption is then constant over time, whereas the variance of consumption levels is increasing or decreasing as the growth rate is positive or negative. With positive growth, the dispersion of consumption is increasing, although that of marginal utility is decreasing. The presence of idiosyncratic uncertainty adds uncorrelated innovations to the Euler equation and will make it more likely that both marginal utilities and consumption disperse over time.

\section{Borrowing Constraints, Buffer Stock Saving, and Earnings Dispersion}

In the simplest model of liquidity-constrained consumers, consumption is equal to earnings and there are neither savings nor assets. In this case, the cross-sectional distribution of consumption is the same as the cross-sectional distribution of earnings, and the two must move together with age. Of course, this is too simple. An inability to borrow does not imply an inability to save, and even those who cannot borrow and have no wish to accumulate will typically have a working balance of cash on hand that acts as a reservoir for emergencies and smooths over short-term fluctuations in earnings. The literature on such "buffer stock" models is reviewed in Deaton (1991); as in Lucas (1980, 1992), liquidity constraints, impatience, and stationary earnings can generate an invariant distribution for consumption in which consumption inequality is constant. (Sufficiently patient consumers want to save, not borrow, and are unaffected by borrowing constraints.) In the invariant distribution, assets follow a renewal process, stocking out from time to time, and the distributions of consumption, earnings, assets, and income are all stationary. Because assets buffer consumption against earnings fluctuations, consumption inequality-in 
the cross section as well as over time-will be less than earnings inequality, which, since assets are small, will be close to income inequality. Apart from the assumption that earnings are stationary, these predictions are close to what we see for Britain and the United States, although the propinquity of consumption inequality to earnings and income inequality in the former remains a puzzle. Since Taiwanese households save so much of their incomes, they are not likely candidates for an explanation whose assumptions preclude long-run accumulation.

Buffer stock models can also accommodate nonstationary earning streams (see Deaton 1991). For example, if the earnings process has a unit root that controls long-run behavior but is also affected by transitory stationary components, liquidity-constrained consumers can use assets to buffer transitory fluctuations in earnings, whereas over the longer run, consumption must adapt to secular changes in earnings. Such models can generate invariant distributions for the ratios of consumption, income, and assets to earnings, so that all four quantities would disperse in parallel, which is close to what happens in the British and American data. (These results apply to the equilibrium invariant distribution, and a much wider range of behavior is possible during the transition into that state.)

Buffer stock models shift the focus for explaining the dispersion of inequality away from consumption and toward earnings. It would take us too far afield in this paper to discuss this much larger topic, but there are several possible explanations: Mincer (1974) predicts increasing inequality in earnings because different individuals choose different amounts of postschool training, screening models have the same implication if the (constant) distribution of abilities is only slowly revealed, and standard human capital models will also predict increasing dispersion of earnings if the rate of return to human capital is different for different individuals. The fact that the within-cohort dispersion of earnings increases with age prevents us from using the theory as a basis for a sharp test between the PIH and the buffer stock models. If we could find a cohort of individuals for whom there is no increase in the dispersion in the cross-section distribution of earnings, a finding of increasing consumption inequality would reject the buffer stock model, and a finding of no increase would reject the PIH.

\section{Insurance and Consumption Inequality}

Another model of consumption that has received a good deal of attention in the recent literature is one in which there exists a complete set of state-contingent securities so that agents can insure themselves 
and remove idiosyncratic consumption risk. Such models have been tested for the United States by Cochrane (1991), Mace (1991), and Altonji, Hayashi, and Kotlikoff $(1991,1992)$, with almost uniformly negative results (see Attanasio and Weber [1992] and Nelson [1994] for a contradiction of Mace's results using the same data) and rather more successfully for Indian villages by Townsend (1994). A complete set of contingent markets implies that the marginal utilities of money $\lambda\left(c_{i t}\right)$ have the factor structure

$$
\lambda\left(c_{i t}\right)=\omega_{i} \gamma_{t} ;
$$

see, for example, Altug and Miller (1990), Altonji et al. (1991), or Deaton (1992, chap. 1) for an exposition.

Given (18), the distribution of log marginal utility is constant over time. Individual consumptions move together as well, with no general increase in their dispersion, although particular functional forms will imply particular patterns for specific measures of inequality. Isoelastic preferences, in particular, imply that the variance of log consumption will be constant over time, something strongly rejected by the data. It is possible, however, to think of examples that can reconcile the data with the model of complete insurance. Steve Davis has drawn to our attention the case in which preferences are not strongly intratemporally separable between leisure and goods, so that leisure also appears on the left-hand side of (18). If individual wage rates (marginal productivities) diverge over time-for example, because different people learn from experience at different rates-then it is socially efficient for the most productive to work more hours and to be compensated by having greater consumption. Even in the full insurance equilibrium, consumption inequality will grow if wage dispersion does so. Heterogeneity in preferences-for example, in rates of time preference or in life cycle taste shifters-could also produce time patterns in the dispersion of consumption, although there is no reason why heterogeneity would necessarily result in increasing dispersion. The results in figure 8 indicate that even after we adjust consumption inequality for dispersion in family size, a possibly important source of heterogeneity, consumption inequality still increases with age.

Models of complete insurance take no account of the problems of information and moral hazard that are likely to prevent their practical realization, and there is a literature that inquires into optimal intertemporal consumption schemes with limited information. In these normative-and typically nondecentralizable-models, such as Thomas and Worrall (1990) and Atkeson and Lucas (1992), the (constrained) socially optimal arrangements involve increasing inequality over time, as in our empirical results, and in contrast to the case of complete insurance. Although these models are useful as bench- 
marks, they are hardly promising candidates for interpreting the evidence. The construction and testing of market models with partial insurance remains an important task for the future.

\section{Conclusions and Further Implications}

Our main purpose in this paper has been to draw attention, first, to the implications of life cycle theory for inequality and, second, to the fact that within-cohort inequality does indeed increase with age, at least for the three countries we have examined. Even if we remain uncertain about the connection between the former and the latter, the fact that inequality increases with age has a number of implications, implications that are made the more interesting by their possible basis in intertemporal theory.

First, consider the implications of the results for inequality in society as a whole. If there are no links between successive generations, if the age distribution of the population remains unchanged, and if the inequality in the distribution of earnings remains constant, consumption and income inequality will also remain constant in the society as a whole, even though inequality is increasing for each age group. The theory is therefore consistent with unchanging or slowly changing inequality in society as a whole, and the data do indeed show extended periods during which inequality is constant (see Levy and Murnane [1992] for the United States). Even when there are changes in aggregate inequality, the results in figures $1-3$ show that they are many times smaller than the changes in inequality associated with aging within given cohorts of people. If there are strong intergenerational links, where bequests are important or in the limit where households are organized into eternal dynasties, the theory predicts widening inequality for society as a whole, at least if no new dynasties are ever created. The failure of such predictions would seem to be evidence against the most extreme versions of such models, but there still remains an important research agenda of building bequest motives into the sort of models examined in this paper and drawing out the implications for the transmission of inequality from older to younger cohorts.

Second, the relationship between age and inequality forges a powerful link between demographic change and the distribution of resources, a link that is largely independent of the theoretical basis of the result. Countries such as Taiwan that have experienced a rapid demographic transition are aging rapidly, so that there are now many more 55-year-olds relative to 25-year-olds than was the case 25 years ago. Since income and consumption are more unequally distributed among the former than the latter, the overall distribution of income 
and consumption will become more unequal, and this is exactly what has happened in Taiwan over the last decade or so. Deaton and Paxson (1993a) derive general results on the relationship between aggregate inequality and population growth in a stable population framework and apply the results to Taiwan.

Third, consumption inequality has implications for aggregation, especially for those attempts to model average consumption as the behavior of an intertemporally efficient representative agent. One defense of applying the theory to aggregate data is that, in the absence of distributional change, functions of averages will behave similarly to averages of functions. The ratio of the average of the logarithms of consumption to the logarithm of the average of consumption is a measure of consumption dispersion, so that when dispersion is increasing, the average of logs will behave quite differently from the logarithm of the average. Indeed, results by Attanasio and Weber (1993) using the British data show that estimates of intertemporal models are quite sensitive to whether or not the aggregation is correctly done.

Fourth, our results have implications for the cross-country relationship between inequality and economic development, first investigated by Kuznets (1955). Kuznets suggested that inequality should rise in the early stages of economic development but should eventually fall as incomes rise, a result that he explained in terms of the urbanization that typically accompanies economic growth. The empirical validity of this inverted $U$-shape has been subject to a great deal of controversy and is far from widely accepted, largely because of the extremely poor quality of international measures of inequality. The results of this paper suggest a different basis for a Kuznets-type relationship. Economic development is eventually accompanied by a demographic transition, from high fertility to low fertility, a transition that eventually redistributes population from young to old. Such a redistribution will tend to lead to widening inequality, at least until the new stable population distribution is established, at which point inequality growth will cease. Of course, income growth may itself affect inequality independently of these demographic effects, with higher growth rates resulting in greater inequality across cohorts.

\section{References}

Altonji, Joseph G.; Hayashi, Fumio; and Kotlikoff, Laurence J. "Risk Sharing, Altruism, and the Factor Structure of Consumption." Working Paper no. 3834. Cambridge, Mass.: NBER, September 1991.

. "Is the Extended Family Altruistically Linked? Direct Tests Using Micro Data." A.E.R. 82 (December 1992): 1177-98. 
Altug, Sumru, and Miller, Robert A. "Household Choices in Equilibrium." Econometrica 58 (May 1990): 543-70.

Atkeson, Andrew, and Lucas, Robert E., Jr. "On Efficient Distribution with Private Information.” Rev. Econ. Studies 59 (July 1992): 427-53.

Atkinson, Anthony B. "On the Measurement of Inequality." J. Econ. Theory 2 (September 1970): 244-63.

Attanasio, Orazio P., and Weber, Guglielmo. "Consumption Growth and Excess Sensitivity to Income: Evidence from US Micro Data." Manuscript. Stanford, Calif.: Stanford Univ.; London: Univ. Coll., April 1992.

- "Consumption Growth, the Interest Rate and Aggregation." Rev. Econ. Studies 60 (July 1993): 631-50.

Campbell, John Y. "Does Saving Anticipate Declining Labor Income? An Alternative Test of the Permanent Income Hypothesis." Econometrica 55 (November 1987): 1249-73.

Chamberlain, Gary. "Panel Data." In Handbook of Econometrics, vol. 2, edited by Zvi Griliches and Michael D. Intriligator. Amsterdam: North-Holland, 1984.

$\rightarrow$ Cochrane, John H. "A Simple Test of Consumption Insurance." J.P.E. 99 (October 1991): 957-76.

Cutler, David M., and Katz, Lawrence F. "Macroeconomic Performance and the Disadvantaged." Brookings Papers Econ. Activity, no. 2 (1991), pp. 1-61.

Deaton, Angus. "Saving and Liquidity Constraints." Econometrica 59 (September 1991): 1221-48.

- Understanding Consumption. Oxford: Clarendon, 1992.

Deaton, Angus, and Paxson, Christina H. "Inequality and Aging: An East Asian Perspective.” Manuscript. Princeton, N.J.: Princeton Univ., Res. Program Development Studies, June 1993. (a)

- "Saving, Growth, and Aging in Taiwan." Working Paper no. 4330. Cambridge, Mass.: NBER, April 1993. (b)

$\rightarrow$ Dooley, Martin D., and Gottschalk, Peter. "Earnings Inequality among Males in the United States: Trends and the Effect of Labor Force Growth." J.P.E. 92 (February 1984): 59-89.

$\rightarrow$ Eden, Benjamin. "Stochastic Dominance in Human Capital." J.P.E. 88 (February 1980): 135-45.

$\rightarrow$ Flavin, Marjorie A. "The Adjustment of Consumption to Changing Expectations about Future Income." J.P.E. 89 (October 1981): 974-1009.

Goodfriend, Marvin: "Information-Aggregation Bias." A.E.R. 82 (June 1992): 508-19.

Grossman, Sanford J., and Shiller, Robert J. "Consumption Correlatedness and Risk Measurement in Economies with Non-traded Assets and Heterogeneous Information.” J. Financial Econ. 10 (July 1982): 195-210.

Hall, Robert E. "The Measurement of Quality Change from Vintage Price Data." In Price Indexes and Quality Change, edited by Zvi Griliches. Cambridge, Mass.: Harvard Univ. Press, 1971.

$\longrightarrow \rightarrow-$ "Stochastic Implications of the Life Cycle-Permanent Income Hypothesis: Theory and Evidence." J.P.E. 86 (December 1978): 971-87.

$\rightarrow$ Kuznets, Simon. "Economic Growth and Income Inequality." A.E.R. 45 (March 1955): 1-28.

$\rightarrow$ Levy, Frank, and Murnane, Richard J. "U.S. Earnings Levels and Earnings Inequality: A Review of Recent Trends and Proposed Explanations." J. Econ. Literature 30 (September 1992): 1333-81. 
Lucas, Robert E., Jr. "Equilibrium in a Pure Currency Economy." Econ. Inquiry 18 (April 1980): 203-20.

. "On Efficiency and Distribution." Econ. J. 102 (March 1992): 233-47.

$\rightarrow$ Mace, Barbara J. "Full Insurance in the Presence of Aggregate Uncertainty." J.P.E. 99 (October 1991): 928-56.

Mincer, Jacob A. Schooling, Experience, and Earnings. New York: Columbia Univ. Press (for NBER), 1974.

$\rightarrow$ Nelson, Julie A. "On Testing for Full Insurance Using Consumer Expenditure Survey Data." J.P.E. 102 (April 1994): 384-94.

Pischke, Jörn-Steffen. "Individual Income, Incomplete Information, and Aggregate Consumption.” Working Paper no. 289. Princeton, N.J.: Princeton Univ., Indus. Relations Sec., 1991.

Republic of China. Report on the Survey of Personal Income Distribution in Taiwan Area of the Republic of China. Taipei: Directorate-General, Budget, Accounting and Statis. Executive Yuan, 1989.

Thomas, Jonathan, and Worrall, Tim. "Income Fluctuation and Asymmetric Information: An Example of a Repeated Principal-Agent Problem." J. Econ. Theory 51 (August 1990): 367-90.

Townsend, Robert M. "Risk and Insurance in Village India." Econometrica 62 (May 1994): 539-91. 